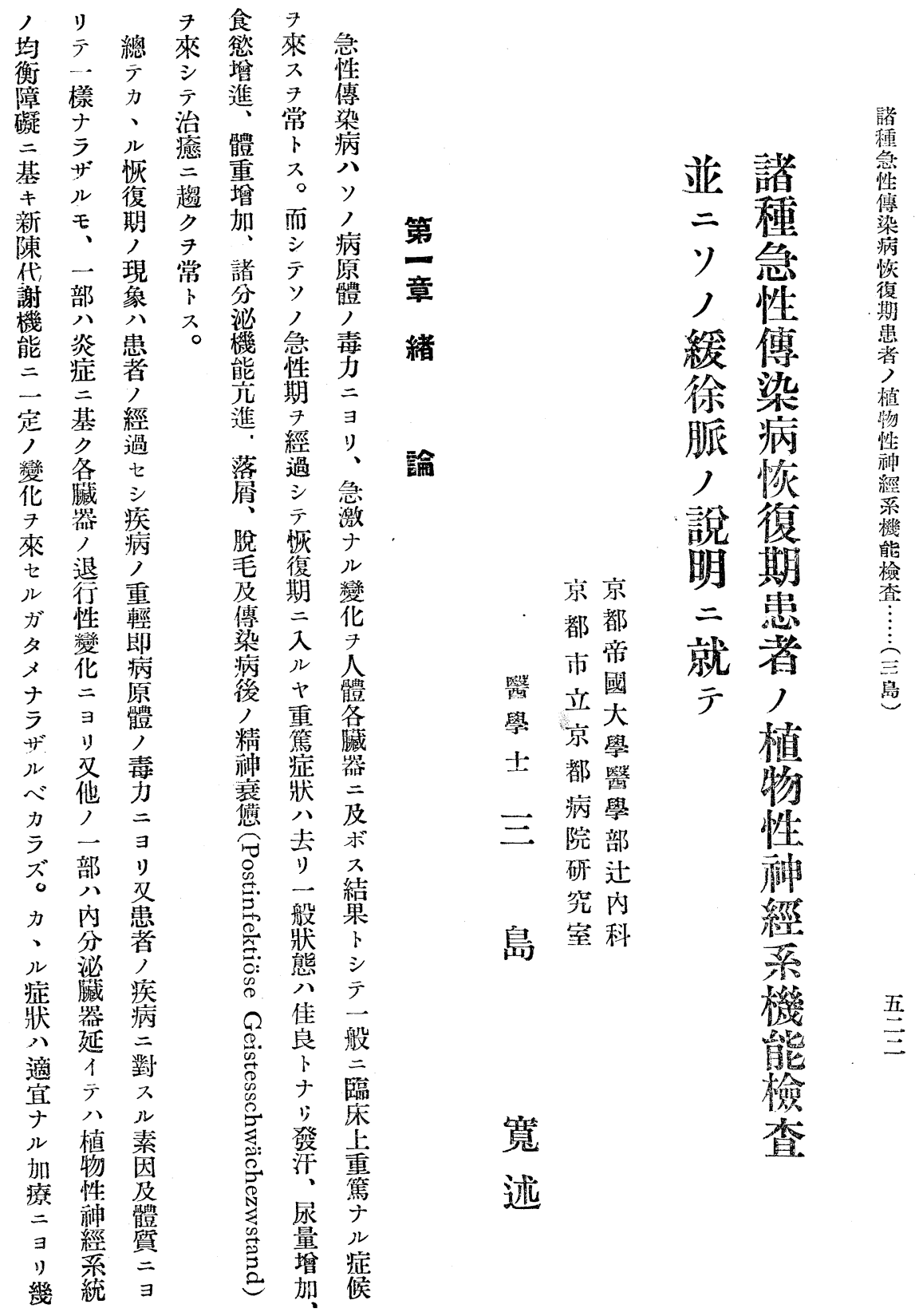




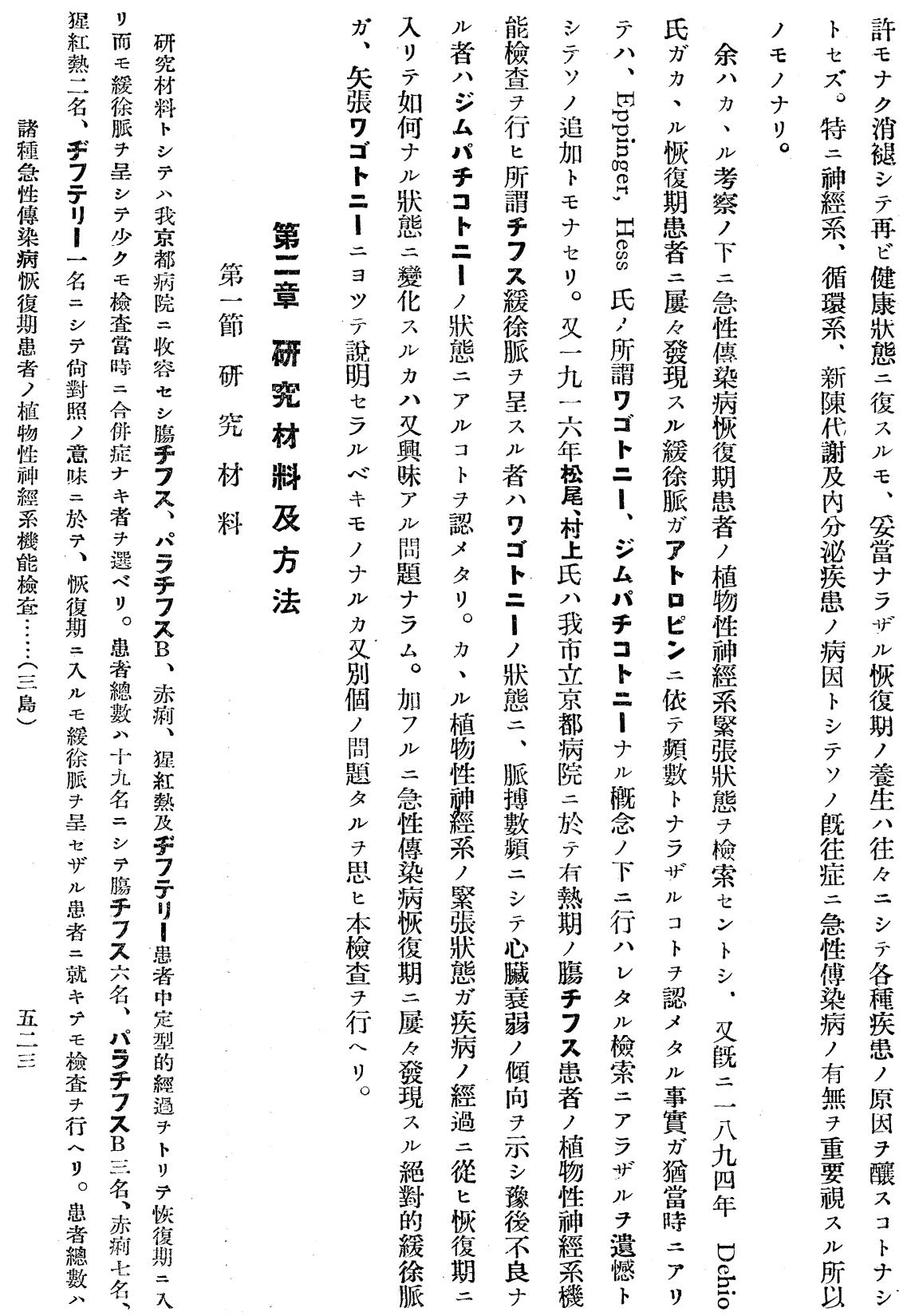




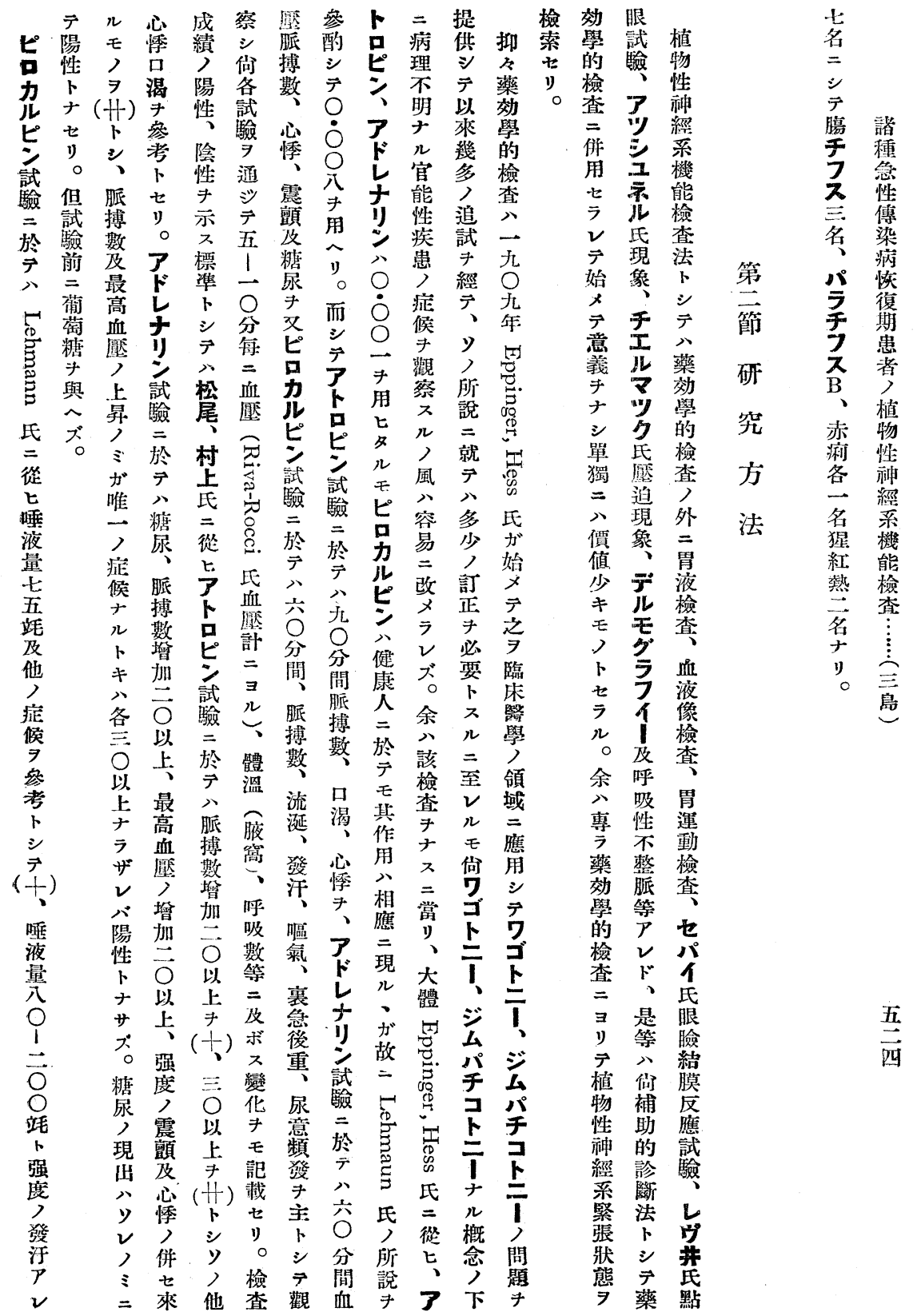




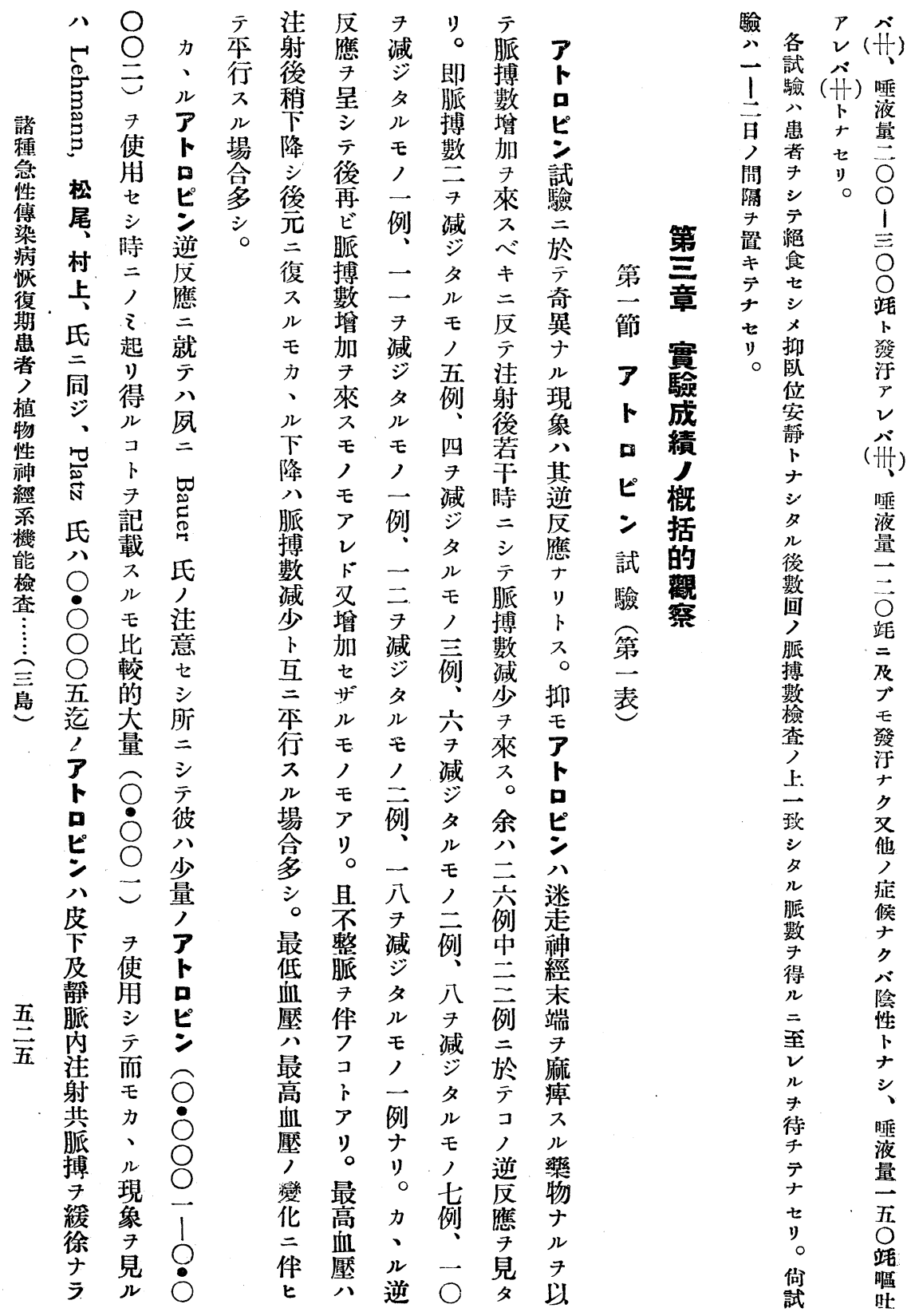


アトロピン試驗(第一表)

\begin{tabular}{|c|c|c|c|c|c|c|c|c|c|c|}
\hline 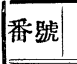 & 姓 & 名 & 性 & 年捽会 & 斷 & 䌉徐胍 & $\begin{array}{l}\text { 脈搏數 } \\
\text { 增加 }\end{array}$ & 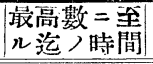 & 口渴 & 心悸 \\
\hline 1 & $\mathrm{~T}$. & $\mathrm{O}$ & $\hat{\delta}$ & 45 & 腸チフス & + & +2 & $30^{\prime}$ & + & - \\
\hline 2 & N. & K. & $q$ & 32 & $" 1$ & + & +14 & $45^{\prime}$ & + & - \\
\hline 3 & $\mathrm{~T}$. & A. & 占 & 28 & "I & + & +2 & $10^{\prime}$ & - & - \\
\hline 4 & $\mathrm{~T}$. & N. & 占 & 31 & "I & + & -8 & $50^{\prime}$ & - & - \\
\hline 5 & T. & N. & $\uparrow$ & 23 & !I & + & +6 & $70^{\prime}$ & - & - \\
\hline 6 & $\mathrm{Y}$. & N. & $\hat{s}$ & 26 & "I & + & +10 & $35^{\prime}$ & - & - \\
\hline 7 & $\mathrm{~T}$. & M. & 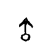 & 17 & パラチフス B & + & +56 & $60^{\prime}$ & + & + \\
\hline 8 & S. & S. & $\hat{\jmath}$ & 20 & "I & + & +14 & $60^{\prime}$ & - & - \\
\hline 9 & $\mathrm{~T}$. & Y. & $\hat{\jmath}$ & 23 & "I & + & +12 & $60^{\prime}$ & + & - \\
\hline 10 & $\mathrm{~K}$. & F. & $\hat{\delta}$ & 19 & "I & - & +16 & $45^{\prime}$ & - & - \\
\hline 11 & $\mathrm{~T}$. & $\mathrm{N}$. & $q$ & 27 & 腸チフス & - & +12 & $45^{\prime}$ & + & - \\
\hline 12 & 'T. & I. & $q$ & 35 & "I & - & +16 & $20^{\prime}$ & + & - \\
\hline 13 & $\mathrm{Y}$. & $\mathrm{H}$. & $\hat{o}$ & 16 & "I & - & +42 & $50^{\prime}$ & + & - \\
\hline 14 & F. & M. & $\hat{s}$ & 41 & 赤 淑 & + & +2 & $70^{\prime}$ & - & - \\
\hline 15 & $\mathrm{~S}$. & J. & + & 21 & " & + & +12 & $55^{\prime}$ & - & - \\
\hline 16 & M. & Y. & $\hat{\delta}$ & 46 & "I & + & +10 & $55^{\prime}$ & - & - \\
\hline 17 & I. & $\mathrm{N}$. & $\hat{\delta}$ & 36 & " & + & -14 & $25^{\prime}$ & + & - \\
\hline 18 & $Z$. & N. & $\hat{s}$ & 32 & " & + & +2 & $70^{\prime}$ & - & - \\
\hline 19 & I. & $\mathrm{K}$. & $\hat{\delta}$ & 17 & " & + & +14 & $60^{\prime}$ & 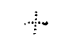 & - \\
\hline 20 & J. & $\mathrm{K}$ & $\hat{\delta}$ & 30 & " & + & +8 & $70^{\prime}$ & + & - \\
\hline 21 & $\mathrm{~K}$. & Y. & $\hat{\delta}$ & 22 & " & - & +2 & $10^{\prime}$ & + & - \\
\hline 22 & K. & T. & $\hat{\delta}$ & 21 & 显 紅 熱 & + & +22 & $90^{\circ}$ & - & - \\
\hline 23 & S. & $\mathrm{Y}$. & $\hat{\delta}$ & 29 & "I & + & +5 & $55^{\prime}$ & 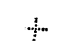 & - \\
\hline 24 & $\mathrm{Y}$. & M. & ㅇ & 16 & "I & - & -18 & $50^{\prime}$ & + & - \\
\hline 25 & G. & M. & $\hat{\delta}$ & 17 & "I & - & +12 & $80^{\prime}$ & - & - \\
\hline 26 & $\mathrm{~K}$. & $\mathrm{H}$. & $\hat{s}$ & 30 & チ゚フテリー & + & +14 & $50^{\prime}$ & + & - \\
\hline
\end{tabular}

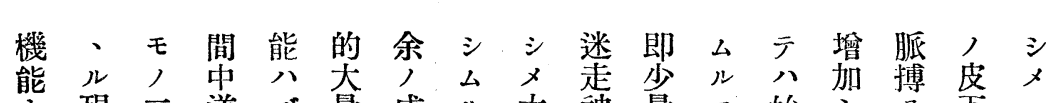

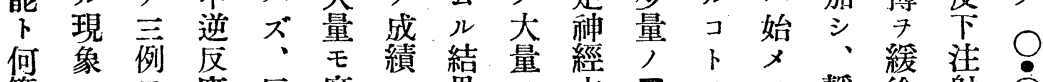

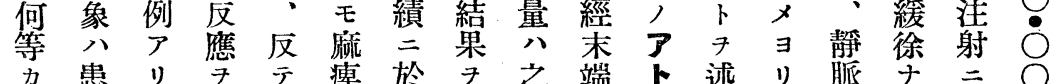

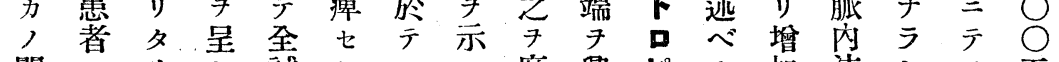

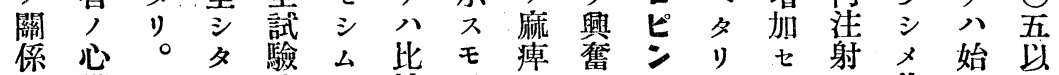

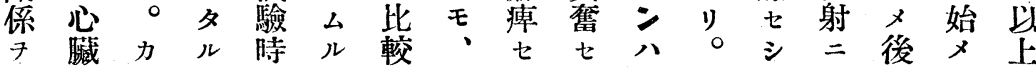




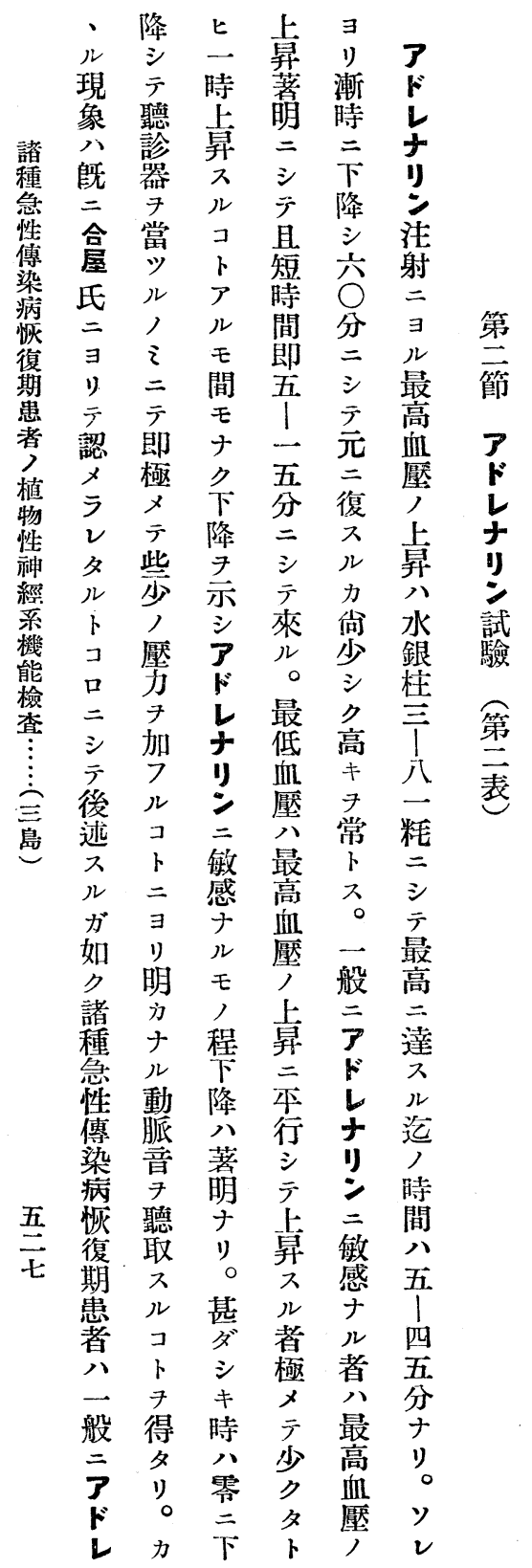

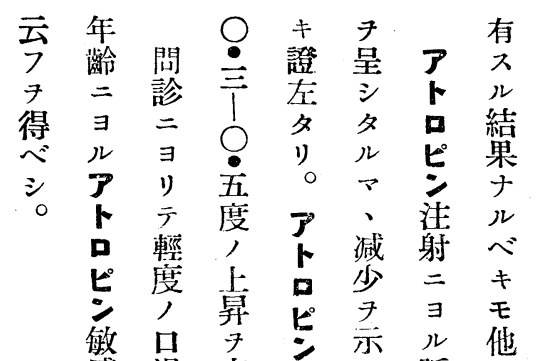

感渴來注不脈日

度 $尹$ 七射 $€$ 搏追

, 認 $\triangleq$, 數 加

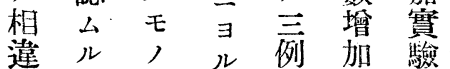

八者最體乃八,

年劣正溫”余上

者較多占脈實

的試兵摶羷 改

少多驗㜢三

キ平終殆增於不

多等架架宁登

正訴浢学文第表

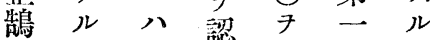

$尹$ 二元婄越表卜

失至蕧ザ至系吕



恐者。モモ无う

于公呼) ’ 如

干殆吸寻多赑

产整当吾兵

今惢度即子

ザ。ボノア最

儿心卡褧

正悸影暴品

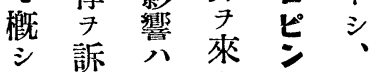

年荠薄示

省者ナモ゙感最

竟僅 ズ范ラ贞

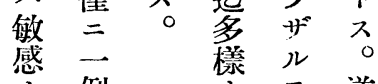

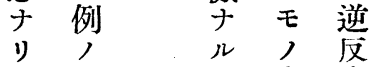

ri E多應 
アトレレナリン試驗 （第二表）

\begin{tabular}{|c|c|c|c|c|c|c|c|c|c|c|c|}
\hline 番號 & 姓 名 & 性 & 年歯命 & 路 & 緩徐脈 & 脈數增加 & 血靨 & 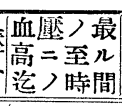 & 心悸 & 震䫓 & 糖尿 \\
\hline 1 & T. O. & $\hat{o}$ & 45 & 勜チフス & + & 24 & 75 & $15^{\prime}$ & + & + & - \\
\hline 2 & N. K. & $q$ & 32 & "I & + & 24 & 22 & $45^{\prime}$ & + & + & - \\
\hline 3 & T. A. & $\hat{s}$ & 28 & " & + & 32 & 33 & $25^{\prime}$ & $H$ & + & - \\
\hline 4 & T. N. & $\hat{s}$ & 31 & " & + & 24 & 68 & $10^{\prime}$ & $H$ & + & - \\
\hline 5 & T. N. & $\hat{\delta}$ & 23 & "I & + & 26 & 68 & $15^{\prime}$ & $H$ & + & - \\
\hline 6 & Y. N. & $\hat{\delta}$ & 26 & " & + & 20 & 34 & $5^{\prime}$ & $H$ & H & - \\
\hline 7 & T. M. & $\uparrow$ & 17 & パラチフス B & + & 22 & 37 & $15^{\prime}$ & + & $H$ & - \\
\hline 8 & S. S. & $\hat{\delta}$ & 20 & "I & + & 32 & 41 & $25^{\prime}$ & $H$ & $H$ & - \\
\hline 9 & T. Y. & $\hat{\delta}$ & 23 & " & + & 30 & 75 & $10^{\prime}$ & \# & $H$ & 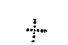 \\
\hline 10 & K. F. & $\hat{\delta}$ & 19 & " & - & 22 & 48 & $10^{\prime}$ & $H$ & + & - \\
\hline 11 & T. N. & 움 & 27 & 膓チフス & - & 20 & 33 & $30^{\prime}$ & + & + & - \\
\hline 12 & 'T. I. & 웅 & 35 & " & - & 24 & 39 & $30^{\prime}$ & + & H & - \\
\hline 13 & Y. H. & $\hat{s}$ & 16 & "I & - & 12 & 48 & $10^{\prime}$ & + & $t$ & - \\
\hline 14 & F. M. & 占 & 41 & 赤 㾥 & + & 12 & 62 & $15^{\prime}$ & $H$ & - & - \\
\hline 15 & S. J, & q & 21 & " & + & 26 & 31 & $20^{\prime}$ & + & + & - \\
\hline 16 & M. Y. & $\hat{\delta}$ & 46 & " & + & 18 & 3 & $10^{\prime}$ & + & + & - \\
\hline 17 & I. N. & $\hat{\delta}$ & 36 & " & + & 26 & 52 & $10^{\prime}$ & H & + & - \\
\hline 18 & Z. N. & $\uparrow$ & 32 & " & + & 26 & 81 & $5^{\prime}$ & H & + & - \\
\hline 19 & I. K. & $\hat{\delta}$ & 17 & "I & + & 30 & 52 & $10^{\prime}$ & $H$ & Ht & - \\
\hline 20 & J. K. & $\hat{\delta}$ & 30 & " & + & 18 & 40 & $20^{\prime}$ & + & 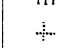 & - \\
\hline 21 & K. Y. & 占 & 22 & " & - & 20 & 47 & $20^{\prime}$ & H & H & - \\
\hline 22 & K. T. & 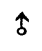 & 21 & 猩紅熱 & + & 30 & 80 & $5^{\prime}$ & H & + & - \\
\hline 23 & S. Y. & s & 29 & $" 1$ & + & 20 & 40 & $10^{\prime}$ & H & + & - \\
\hline 24 & Y. M. & q & 16 & " & - & 20 & 8 & $10^{\prime}$ & + & + & - \\
\hline 25 & G. M. & $\hat{\sigma}$ & 17 & " & - & 20 & 30 & $20^{\prime}$ & + & + & - \\
\hline 26 & K. H. & $\hat{s}$ & 30 & チフテリー & + & 30 & 47 & $20^{\prime}$ & + & + & - \\
\hline
\end{tabular}

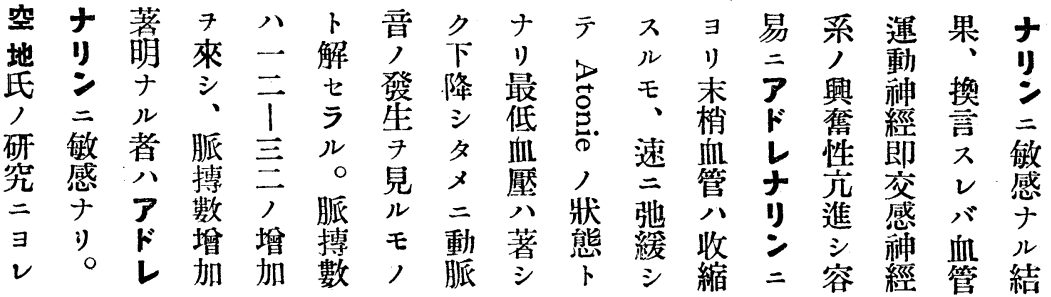




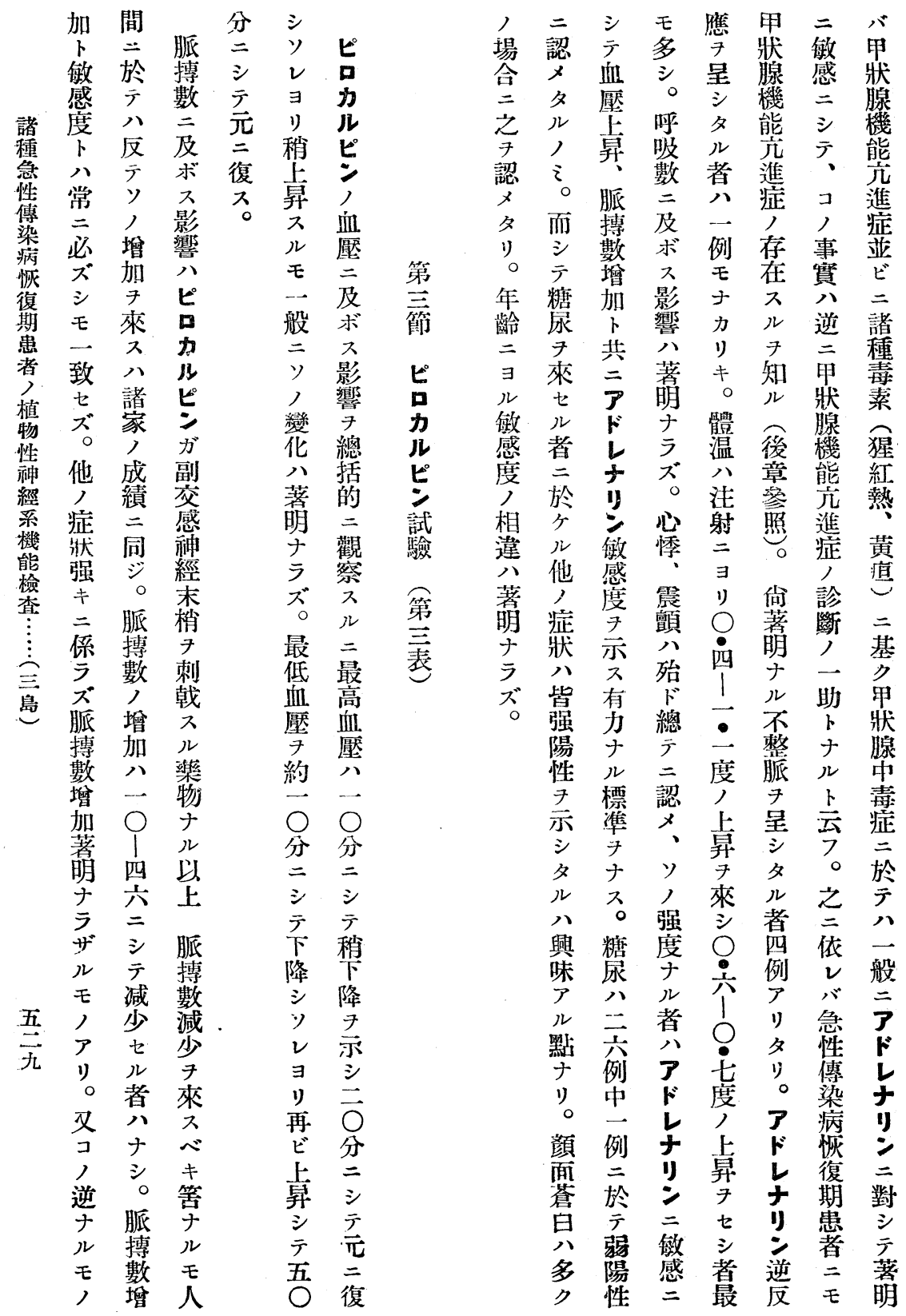


ピロカルピン試驗（第三表）

\begin{tabular}{|c|c|c|c|c|c|c|c|c|c|}
\hline 番號 & 姓：名 & 性 & 年瀜合 & 斷 & $\begin{array}{l}\text { 緩徐 } \\
\text { 脈 } \\
\end{array}$ & $\begin{array}{l}\text { 流涎 } \\
\text { c.c. }\end{array}$ & 發汗 & \begin{tabular}{|l|} 
脈搏數 \\
量加
\end{tabular} & 其他 つ症狀 \\
\hline 1 & T. O. & $\hat{\jmath}$ & 45 & 腸チフス & + & 40 & + & 20 & \\
\hline 2 & N. K. & q & 32 & "I & + & 160 & H & 12 & 嘔氣尿意頻發 \\
\hline 3 & T. A. & $\hat{f}$ & 28 & " & + & 170 & $H$ & 29 & 尿意頻發裏急後重 \\
\hline 4 & T. N. & $\hat{\delta}$ & 31 & " & + & 80 & $H$ & 32 & 尿意頻發 \\
\hline 5 & T. N. & $\hat{f}$ & 23 & "I & + & 70 & + & 42 & 嘔昳 \\
\hline 6 & Y. N. & $\hat{s}$ & 26 & " & + & 80 & H & 10 & 変急後重, 尿意頻發 \\
\hline 7 & T. M. & $\hat{\delta}$ & 17 & パラチフス B & $3+$ & 80 & + & 14 & 裏急後重 \\
\hline 8 & S. S. & $\hat{\delta}$ & 20 & $" 1$ & + & 50 & $H$ & 30 & \\
\hline 9 & T. Y. & $\hat{\delta}$ & 23 & " & + & 170 & $H$ & 22 & 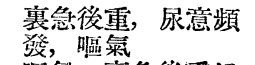 \\
\hline 10 & K. F. & $\hat{\delta}$ & 19 & "I & - & 120 & H & 26 & 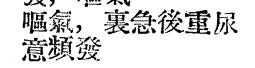 \\
\hline 11 & T. N. & ㅇ & 27 & 笏チフス & - & 20 & - & 32 & \\
\hline 12 & T. I. & ㅇ & 35 & " & - & 145 & $H$ & 16 & 嘔氯, 尿意頻發 \\
\hline 13 & Y. H. & $\uparrow$ & 16 & " & - & 183 & $H t$ & 36 & 尿意頻發 \\
\hline 14 & F. M. & $\hat{s}$ & $4: 1$ & 赤瘚 & + & 115 & H & 14 & \\
\hline 15 & S. J. & 운 & 21 & " & $t$ & 85 & + & 46 & 尿意頻登 \\
\hline 16 & M. Y. & $\hat{\delta}$ & 46 & "I & + & 痕跡 & + & 10 & \\
\hline 17 & I. N. & $\hat{s}$ & 36 & "I & + & 275 & HH & 20 & 嘔吐沓意頻發裏急 \\
\hline 18 & Z. N. & $\hat{\jmath}$ & 32 & " & + & 110 & Ht & 30 & 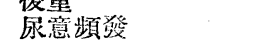 \\
\hline 19 & I. K. & $\hat{f}$ & 17 & " & + & 123 & H & 28 & 尿意頻發 \\
\hline 20 & J. K. & $\hat{\delta}$ & 30 & " & + & 100 & Ht & 20 & 尿意頻發 \\
\hline 21 & K. Y. & $\hat{s}$ & 22 & " & - & 100 & H & 12 & 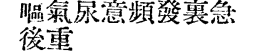 \\
\hline 22 & K. T. & $\hat{s}$ & 21 & 猩 紅 熱 & + & 120 & Ht & 22 & 尿意頻發 \\
\hline 23 & S. Y. & $\hat{\delta}$ & 29 & $" 1$ & + & 106 & H & 32 & 尿意頻發 \\
\hline 24 & Y. M. & ㅇ & 16 & "I & - & 183 & H & 30 & 嘔 氣 \\
\hline 25 & G. M. & $\hat{s}$ & 17 & $"$ & - & 163 & \# & 16 & \\
\hline 26 & K. H. & 占 & 30 & チフテリー & + & 140 & $H$ & 22 & 腹痛, 蠕動 \\
\hline
\end{tabular}

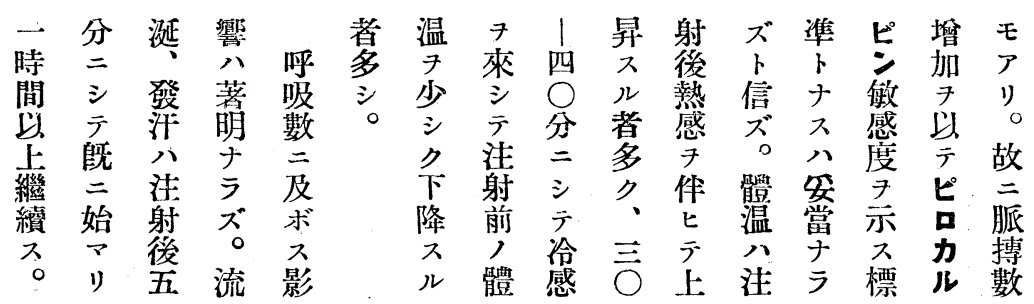




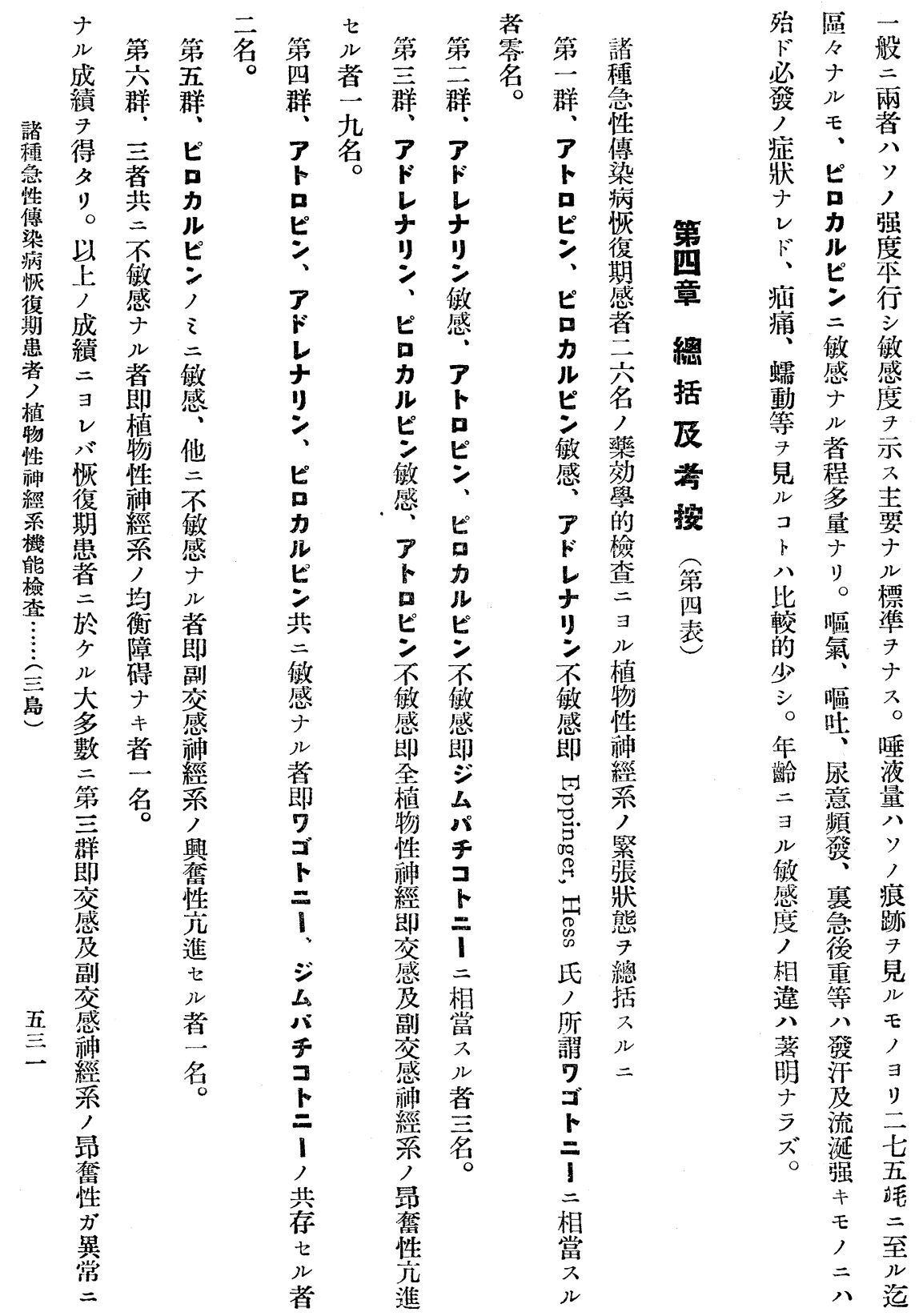




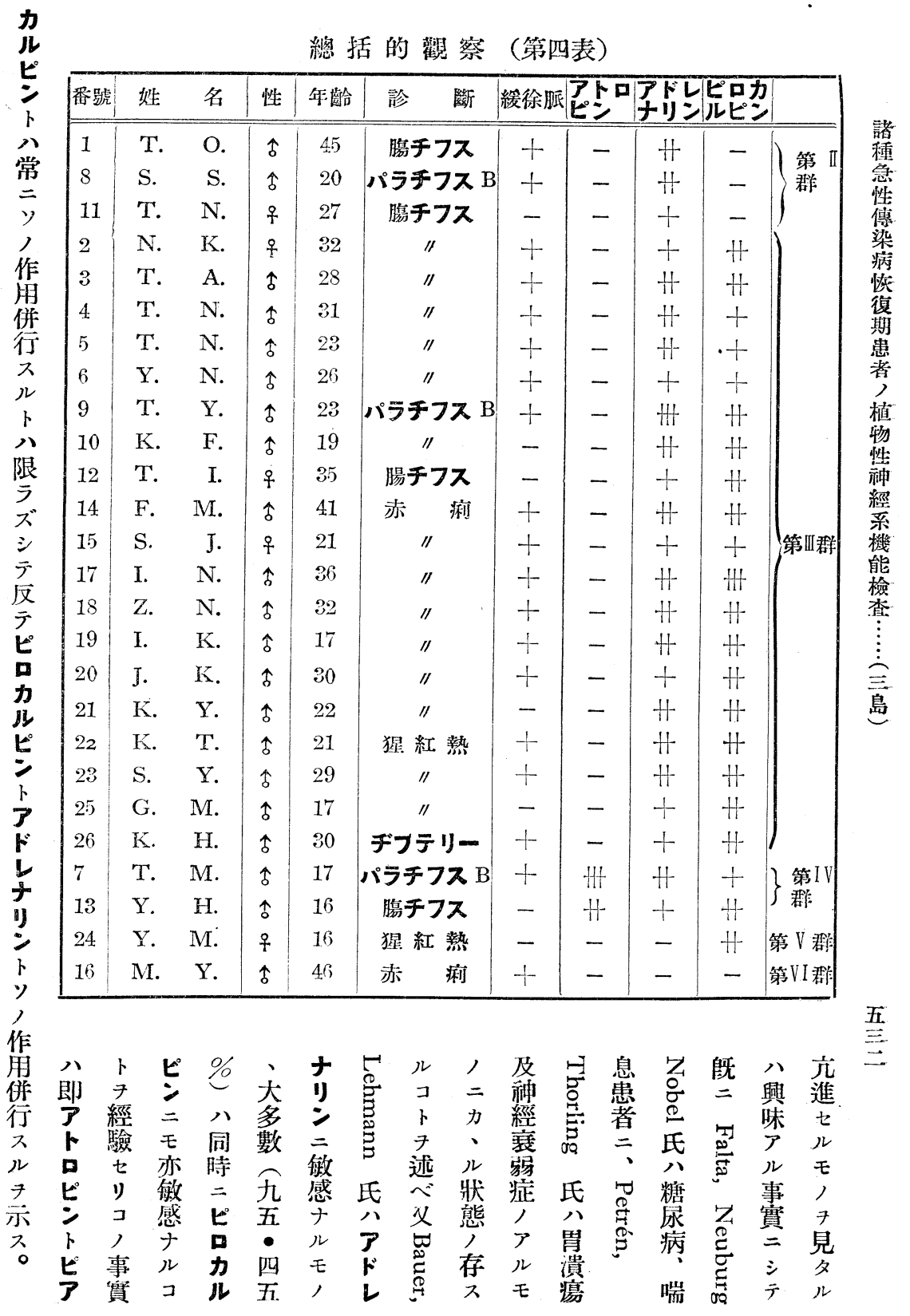




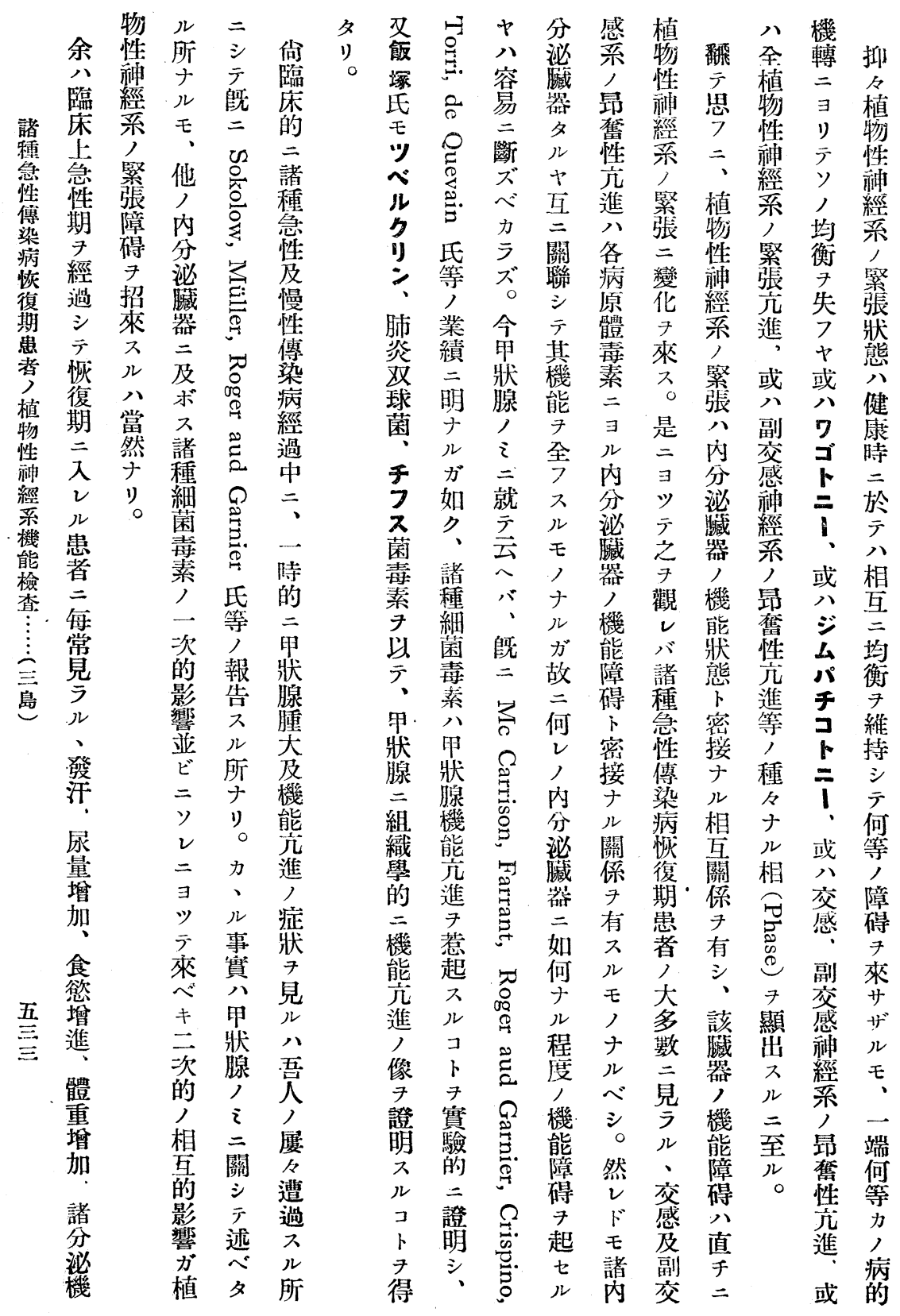




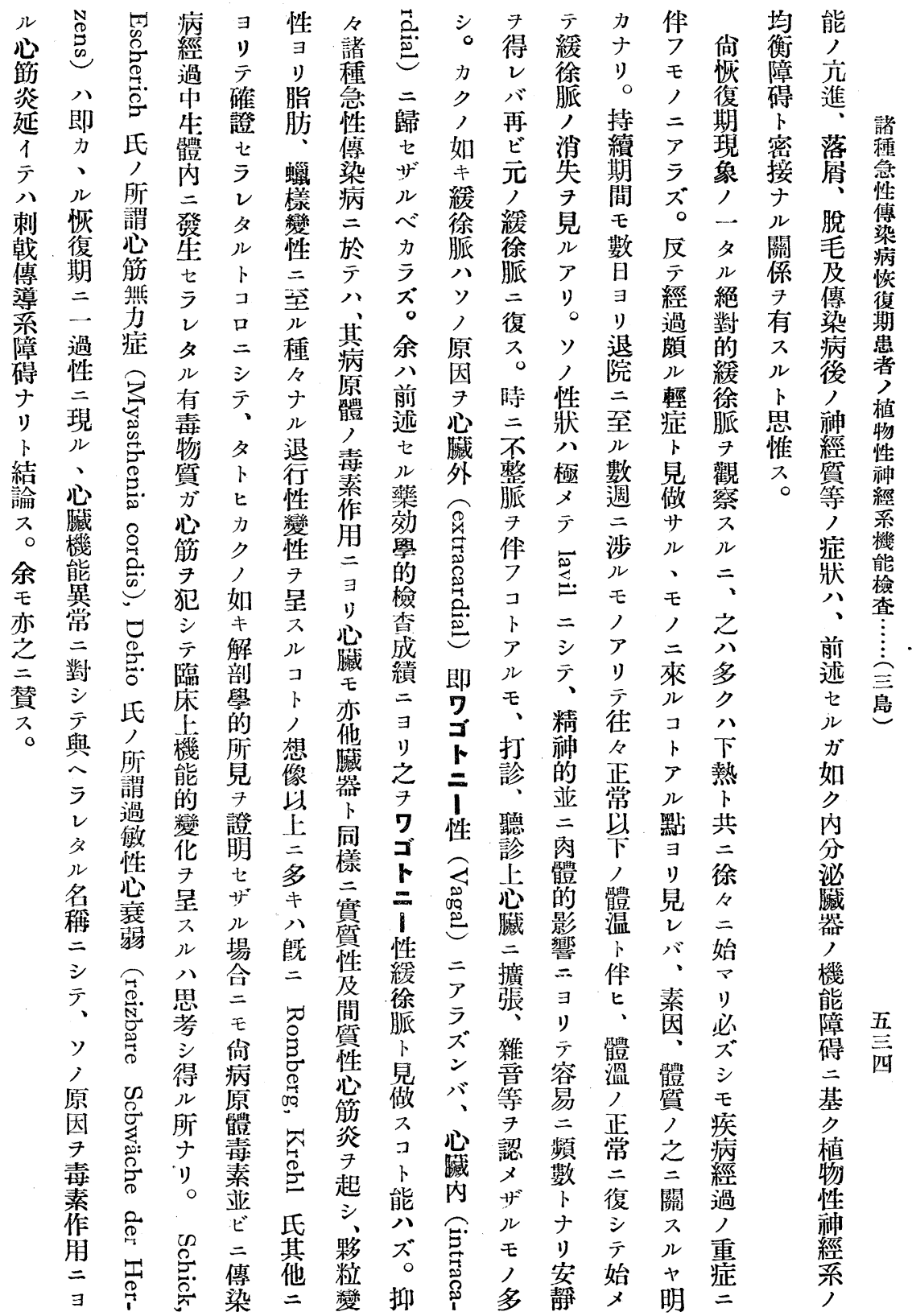




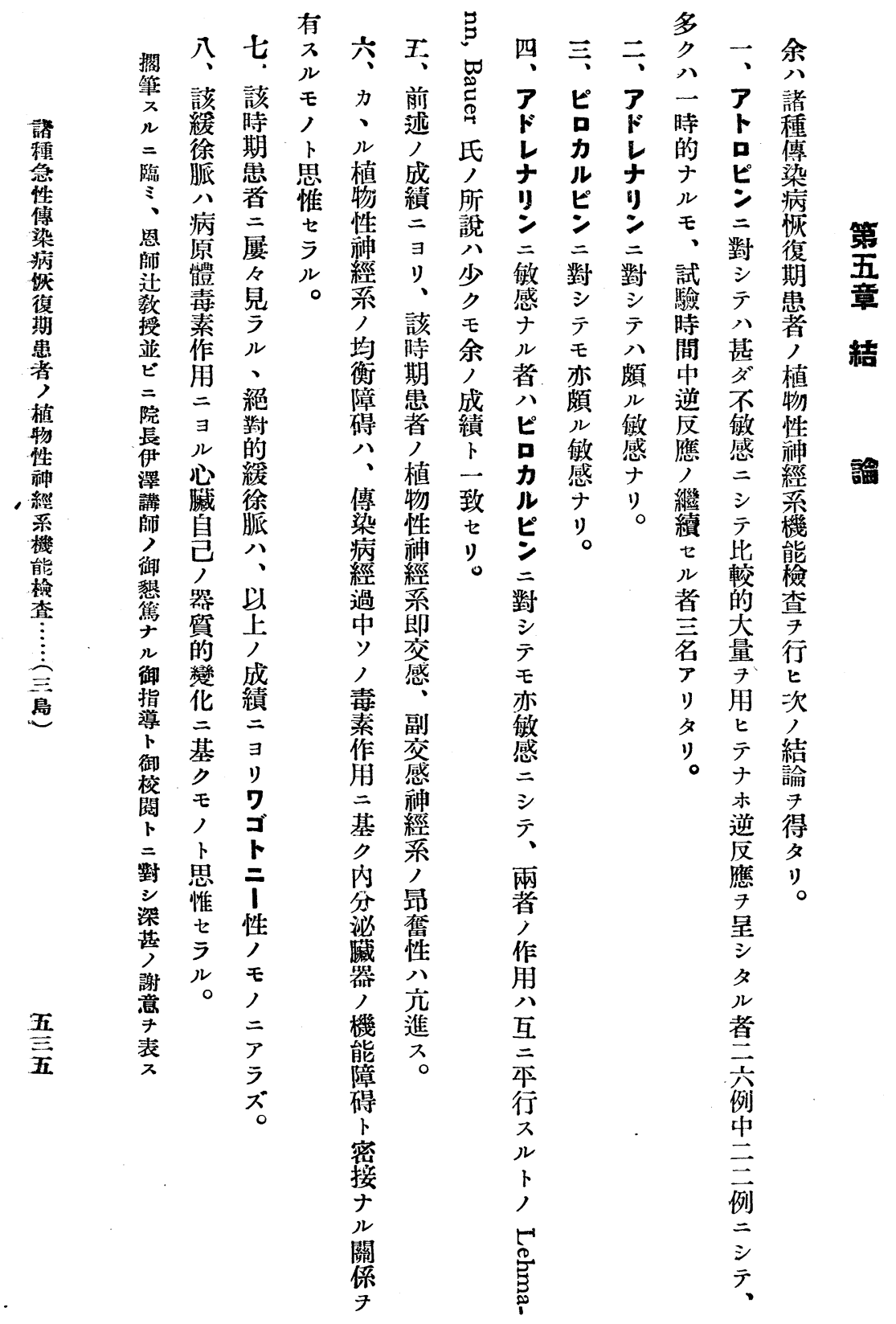




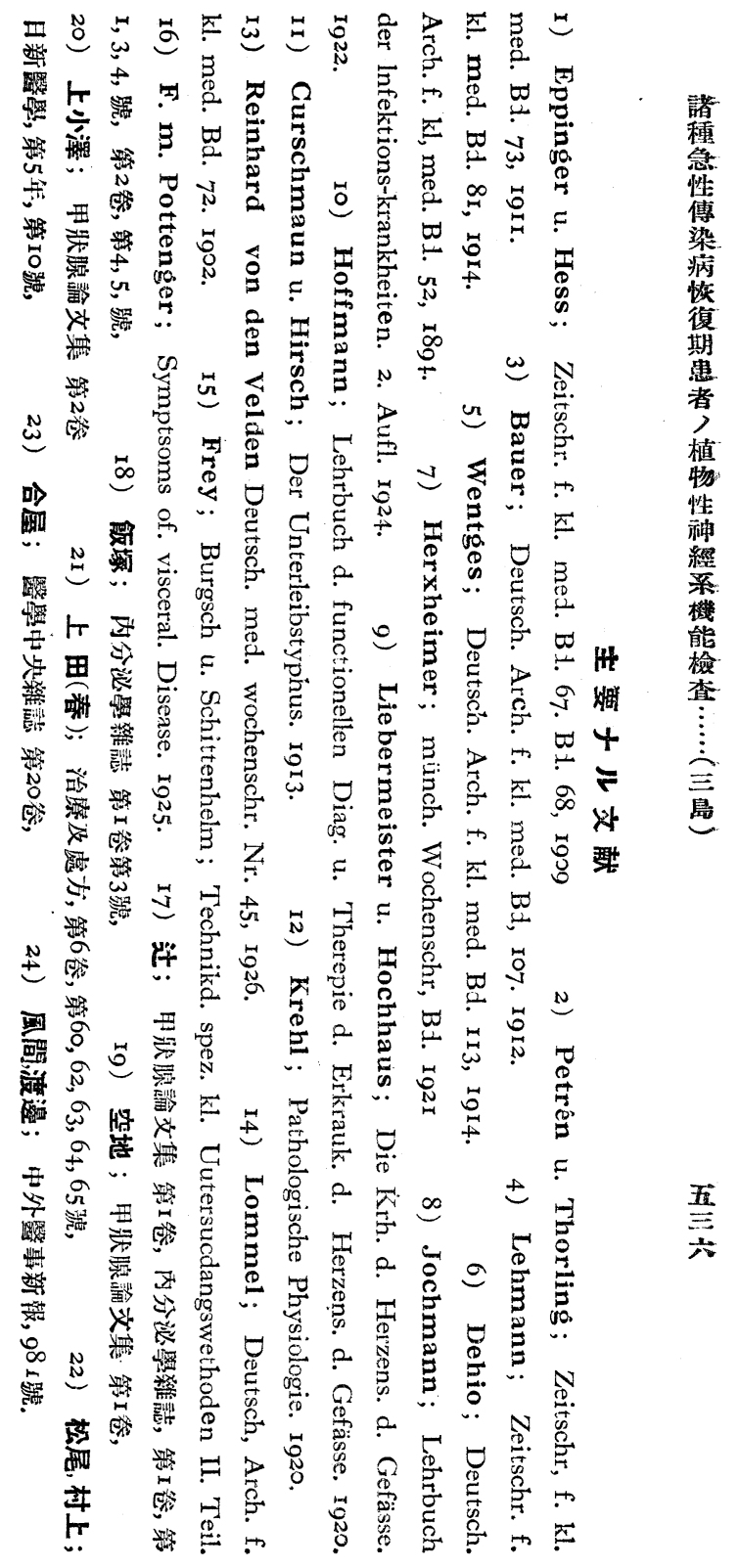


der N-Stoffwechsel deutlich erhöht, womit natürlich die Gesamtstickstoff-, Ammoniakstickstoff- und Kreatinstickstoffausscheidung im Harne gleichzeitig vermehrt sind.

Die Insulinbehandlung, die sich auf 5 bis 20 Tage erstreckte, , rief bei 5 Fällen von Morbus Basedowii und Hyperthyreose, welche Glykosurie oder niedrige Zuckertoleranz zeigten, Herabsetzung des erhöhten N-Stoffwechsels hervor, wodurch sich die Gesamtstickstoff-, Ammoniakstickstoff-und Kreatinstickstoffausscheidung deutlich verminderten. Bei I Fall jedoch ohne niedrige Zuckertoleranz zeigte sich vielmehr eine weitere Erhöhung des N-Stoffwechsels, wenn auch leichten Grades.

Während man bei kurzer Insulinbehandlung keine nennenswerte Besserung bemerkte, übte eine lange Behandlung dagegen einen günstigen Einfluss bei den Fällen mit Glykosurie oder niedriger Zuckertoleranz aus, während bei dem Fall ohne niedrige Zuckertoleranz kein Erfolg zu sehen war. Der günstige Einfluss zeigte sich darin, dass das Körpergewicht zunahm, der Handtremor verschwand, die Pulszahl sich verminderte, die Schweisssekretion abnahm, verschiedene subjektive Beschwerden gelindert wurden u. s. w. Struma und verschiedene Augensymptome zeigten jedoch keine Veränderung. Was die Besserung dieser klinischen Symptome infolge Insulinbehandlung anbelangt, so kommt jedoch dabei in Frage, ob dabei die Insulininjektion selbst oder die lange körperliche und psychische Ruhe die Hauptrolle spielte.

(Autoreferat.)

\author{
UEBER DIE FUNKTIONSPRÜFUNG DES VEGETATIVEN \\ NERVENSYSTEMS BEI REKONVALESZENTEN \\ NACH AKUTEN INFEKTIONSKRANKHEITEN \\ UND ERKL $\ddot{R}$ RNG DER DABEI AUFTRE- \\ TENDEN ABSOLUTEN BRADYCARDIE.
}

Von 
Dr. H. Mishima.

(Aus der I. Med. Klinik d. Kaiserl Univ. zu Kyoto.

Direktor: Prof. K. Tsuji. M. D.)

(Diese Arbeit wurde durch freundliche Unterstützung des Direktors des städtischen Krankenhauses zu Kyoto, Dr. Med. T. Isawa, ausgeführt.)

Viele verschiedene Erscheinungen im Rekonvaleszenzstadium nach akuten Infektionskrankheiten, wie Neigung zum Schwitzen, Vermehrung der Harnmenge, Abschuppung, Haarausfall und postinfektiöse Geistesschwäche,sind Zeichen der Gleichgewichts-und Funktionsstörungenirn vegetativen Nervensystem. Der Verfasser hat nur an den Rekonvaleszenten nach akuten Infektionskrankheiten die Funktionen des vegetativen Nervensystems ausschlisslich pharmakodynamisch geprüft. Sehr interessant erschien Ihn, dass häufig absolute Bradycardie in diesem Stadium auftritt, die nach Dehio durch Atropin nicht frequent wird. Leider wurden damals diese Untersuchungen nicht unter dem Gesichtspunkte der Vagotonie u. Sympathikotonie ausgeführt. Verfasser hat daher seine Rekonvaleszenten in zwei Gruppen geteilt, eine mit Bradycardie, die andere ohne Bradycardie, und an beiden Gruppen seine Versuche vorgenommen.

Die Resultate waren die folgenden:

Die Rekonvaleszenten nach akuten Infektionskrankheiten waren unempfindlich gegen Atropin, und es zeigte sich auf grosse Dosen hin vorübergehend bei 22 unter 26 Fällen diei nverse Wirkung des Atropin und zwar bei 3 Fällen davon dauernd. Die Rekonvalezenten waren sehr empfindlich gegen Adrenalin. Nach Dr. Sorachi sind die Hyperthyreosen im allgemeinen gegen Adrenalin empfindlich, und diese Tatsache kann zur Diagnose der Hyperthyreose benutzt werden.

Danach glaube ich, dass sich unter den Rekonvaleszenten Hyperthyreoidismus vorfindet. Auch gegen Pilocarpin waren die Rekonvaleszenten empfindlich. Es ist bemerkenswert, dass die Wirkung des 
Atropins u. Pilocarpins, wie schon Bauer u. Lehmann konstatierten, miteinander nicht parallel gehen, wohl aber die Wirkung des Adrenalins u. des Pilocarpins. Diese Prüfung zeigt, wie schon erwähnt, dass sich bei den Rekonvaleszenten nach akuten Infektionskrankheiten die Erregbarkeit des vegetativen Systems erhöht.

Solche Gleichgewichtsstörungen im vegetativen Nervensystem stehen in engerer Beziehung einerseits zum endokrinen Drüsensystem, anderseits zu den pathologischen Veränderungen der Gewebe durch die akuten Infektionskrankheiten.

Die Genese der in diesem Stadium häufig auftretenden absoluten Bradykardie ist nicht vagal, sondern ist nach Meinung des Verfassers Reizleitungsstörung infolge infektiöser Myocarditis aufzufassen.

(Autoreferat.)

\section{UEBER DIE PATHOLOGISCHEN VERÄNDERUNGEN}

DER EPITHELKÖRPERCHEN BEI DEN SEKTIONSFÄLLEN, WO SICH IM KNOCHENSYSTEM HOCHGRADIGE METASTASEN VON BÖSARTIGEN GESCHWÜLSTEN VORFINDEN.

Von

Dr. K. Kurokawa.

(Aus dem pathologisch-bakteriologischen Institut der KeioUniversität zu Tokyo. Direktor: Prof. Dr. S. Kusama.)

Ich beobachtete bei den folgenden 3 Sektionsfällen fast die gleichen pathologischen Veränderungen an den Epithelkörperchen.

Fall I, Mann, $5^{8}$ Jahre.

Das primäre Spindelzellensarkom des Oberarms metastasierte und zerstörte fast alle Knochen. Durchschnittsgrösse der 4 Epithelkörper- 